\title{
On the distribution of winners' scores in a round-robin tournament
}

\author{
Yaakov Malinovsky * \\ Department of Mathematics and Statistics \\ University of Maryland, Baltimore County, Baltimore, MD 21250, USA
}

January 14, 2022

\begin{abstract}
In a classical chess round-robin tournament, each of $n$ players wins, draws, or loses a game against each of the other $n-1$ players. A win rewards a player with 1 points, a draw with $1 / 2$ point, and a loss with 0 points. We are interested in the distribution of the scores associated with ranks of $n$ players after $\left(\begin{array}{l}n \\ 2\end{array}\right)$ games, i.e. the distribution of the maximal score, second maximum, and so on. The exact distribution for a general $n$ seems impossible to obtain; we obtain a limit distribution.
\end{abstract}

Keywords: Complete graph, extremes, negative correlation, Poisson approximation, total variation distance

MSC2020: 62G32; 05C20

\section{Introduction}

In a classical chess round-robin tournament, each of $n$ players wins, draws, or loses a game against each of the other $n-1$ players. A win rewards a player with 1 points, a draw with $1 / 2$ point, and a loss with 0 points. Denoting by $X_{i j}$ the score of the player $i$ after the game with the player $j, j \neq i$, in this article, we consider the following model:

\section{Model M:}

For $i \neq j, X_{i j}+X_{j i}=1, X_{i j} \in\{0,1 / 2,1\}$; we assume that all players are equally strong, i.e. $P\left(X_{i j}=1\right)=P\left(X_{j i}=1\right)$, and that the probability of a draw is the same

*email: yaakovm@umbc.edu 
for all games, denoted by $p=P\left(X_{i j}=1 / 2\right)$. We also assume that all $\left(\begin{array}{l}n \\ 2\end{array}\right)$ pairs of scores $\left(X_{12}, X_{21}\right), \ldots,\left(X_{1 n}, X_{n 1}\right), \ldots,\left(X_{n-1, n}, X_{n, n-1}\right)$ are independent.

Let $s_{i}=\sum_{j=1, j \neq i}^{n} X_{i j}$ be a score of the player $i(i=1, \ldots, n)$ after playing with $n-1$ opponents. We use a standard notation and denote by $s_{(1)} \leq s_{(2)} \leq \ldots \leq s_{(n)}$ the order statistics of the random variables $s_{1}, s_{2}, \ldots, s_{n}$, and further denote normalized scores (zero expectation and unit variance) by $s_{1}^{*}, s_{2}^{*}, \ldots, s_{n}^{*}$ with the corresponding order statistics $s_{(1)}^{*} \leq s_{(2)}^{*} \leq \cdots \leq s_{(n)}^{*}$.

For the case where there are no draws, i.e. $X_{i j} \in\{0,1\}, X_{i j}+X_{j i}=1, p_{i j}=P\left(X_{i j}=\right.$ 1) $=\frac{1}{2}$, Huber (1963) proved that

$$
s_{(n)}^{*}-\sqrt{2 \log (n-1)} \rightarrow 0
$$

in probability as $n \rightarrow \infty$ (see also Moon (2013)), where $\log (x)$ is the $\operatorname{logarithm}$ of $x$, to base $e$. The main step in his proof was establishing the following inequality (Lemma 1 in Huber (1963)):

$$
P\left(s_{1}<k_{1}, \ldots, s_{m}<k_{m}\right) \leq P\left(s_{1}<k_{1}\right) \cdots P\left(s_{m}<k_{m}\right)
$$

for any probability matrix $\left(p_{i j}\right)$ and any numbers $\left(k_{1}, \ldots, k_{m}\right), m \leq n$.

Malinovsky and Moon (2021) extended Huber's lemma to a large class of discrete distributions of $X_{i j}$ and showed that for generalizations of round-robin tournaments, this extension implies convergence in probability of the normalized maximal score. Model M is a particular case of such generalizations.

Ross (2021) considered a tournament model where $X_{i j} \sim \operatorname{Bin}\left(n_{i j}, p_{i j}\right)$, proved that Huber's type lemma holds for that model, and identified some bounds on the distribution of the number of wins for the winning team. The results from Ross (2021) turned out to be essential in the proof of the main result in this work.

In this work, we are interested in the marginal distribution of the scores associated with the ranks of $n$ players after $\left(\begin{array}{l}n \\ 2\end{array}\right)$ games under Model M, where rank 1 is the winner's rank, rank 2 is the second best, and so on. This means that we are interested in finding the marginal distribution of $s_{(i)}$. The exact distribution for a general $n$ seems impossible to obtain; we obtain a limit distribution, and demonstrate it with the three best scores in Model M. 


\section{Main Result}

Under Model M, we have the following properties of the scores $s_{1}, s_{2} \ldots, s_{n}$ that satisfy $s_{1}+s_{2}+\cdots+s_{n}=n(n-1) / 2$ :

(a) $E_{n}=E\left(s_{1}\right)=(n-1) / 2, \quad \sigma_{n}=\sigma\left(s_{1}\right)=\sqrt{(n-1)(1-p) / 4}$,

(b) $\rho_{n}=\operatorname{corr}\left(s_{1}, s_{2}\right)=-1 /(n-1)$,

(c) The random variables $s_{1}, s_{2}, \ldots, s_{n}$ are exchangeable for the fixed $n$.

The normalized scores $s_{1}^{*}, s_{2}^{*}, \ldots, s_{n}^{*}$ are exchangeable random variables for the fixed $n$, i.e., n-exchangeable or finite exchangeable. Their distribution depends on $n$, and their correlation is a function of $n$. Therefore, if they are a segment of the infinite sequence $s_{1}^{*}, s_{2}^{*}, \ldots$, then they are not exchangeable, i.e., not infinite exchangeable.

Let $I_{j}^{(n)}=I\left(s_{j}^{*}>x_{n}(t)\right)$, where we choose $x_{n}(t)=a_{n} t+b_{n}$, where

$$
a_{n}=(2 \log n)^{-\frac{1}{2}}, \quad b_{n}=(2 \log n)^{\frac{1}{2}}-\frac{1}{2}(2 \log n)^{-\frac{1}{2}}(\log \log n+\log 4 \pi) .
$$

Set $S_{n}=I_{1}^{(n)}+I_{2}^{(n)}+\cdots+I_{n}^{(n)}$.

We prove the following result.

Theorem 1. For $p=0$ or $p \in[1 / 3,1)$ and a fixed value of $k$,

$$
\lim _{n \rightarrow \infty} P\left(S_{n}=k\right)=e^{-\lambda(t)} \frac{\lambda(t)^{k}}{k !}, \lambda(t)=e^{-t}
$$

Proof. (Theorem 1) The result follows from Assertions presented below. Set

$$
\pi_{i}^{(n)}=P\left(I_{i}^{(n)}=1\right), \quad W_{n}=\sum_{i=1}^{n} I_{i}^{(n)}, \quad \lambda_{n}=E\left(W_{n}\right)=\sum_{i=1}^{n} \pi_{i}^{(n)}
$$

\section{Assertion 1.}

$d_{T V}\left(L\left(W_{n}\right), \operatorname{Poi}\left(\lambda_{n}\right)\right) \leq \frac{1-e^{\lambda_{n}}}{\lambda_{n}}\left(\lambda_{n}-\operatorname{Var}\left(W_{n}\right)\right)=\frac{1-e^{\lambda_{n}}}{\lambda_{n}}\left(\sum_{i=1}^{n}\left(\pi_{i}^{(n)}\right)^{2}-\sum_{i \neq j} \operatorname{Cov}\left(I_{i}^{(n)}, I_{j}^{(n)}\right)\right)$

where $d_{T V}\left(L\left(W_{n}\right), \operatorname{Poi}\left(\lambda_{n}\right)\right)$ is the total variation distance between distributions of $W_{n}$ and Poisson distribution with mean $\lambda_{n}$. 


\section{Assertion 2.}

$$
\pi_{1}^{(n)}=P\left(s_{1}^{*}>x_{n}(t)\right) \sim 1-\Phi\left(x_{n}(t)\right)
$$

where $c_{n} \sim k_{n}$ means $\lim _{n \rightarrow \infty} c_{n} / k_{n}=1$.

\section{Assertion 3.}

$$
\lim _{n \rightarrow \infty} n \pi_{1}^{(n)}=\lim _{n \rightarrow \infty} n P\left(s_{1}^{*}>x_{n}(t)\right)=\lambda(t)=e^{-t}
$$

\section{Assertion 4.}

$$
\lim _{n \rightarrow \infty} n^{2}\left(P\left(s_{1}^{*}>x_{n}(t), s_{2}^{*}>x_{n}(t)\right)\right)=\lambda(t)^{2}=e^{-2 t}
$$

In our case, since $s_{1}^{*}, \ldots, s_{n}^{*}$ are identically distributed, $\sum_{i=1}^{n}\left(\pi_{i}^{(n)}\right)^{2}=n P\left(s_{1}^{*}>x_{n}\right) P\left(s_{1}^{*}>x_{n}\right)$, and $\sum_{i \neq j} \operatorname{Cov}\left(I_{i}^{(n)}, I_{j}^{(n)}\right)=n(n-1)\left[P\left(s_{1}^{*}>x_{n}(t), s_{2}^{*}>x_{n}(t)\right)-P\left(s_{1}^{*}>x_{n}(t)\right) P\left(s_{2}^{*}>x_{n}(t)\right)\right]$. Hence, from (A2) and (A3) it follows that

$$
\lim _{n \rightarrow \infty} \sum_{i=1}^{n}\left(\pi_{i}^{(n)}\right)^{2}=0
$$

and from (A3) and (A4) it follows that

$$
\lim _{n \rightarrow \infty} \sum_{i \neq j} \operatorname{Cov}\left(I_{i}^{(n)}, I_{j}^{(n)}\right)=0 .
$$

Then, from (F1) and (F2) it follows that $\lim _{n \rightarrow \infty} d_{T V}\left(L\left(W_{n}\right), \operatorname{Poi}\left(\lambda_{n}\right)\right)=0$, and this completes the proof of Theorem 1 .

Proof. (Assertion 1). If $p=P\left(X_{i j}\right)=0$ then $X_{i j}$ has Bernoulli distribution which is log-concave; or if $p \geq 1 / 3$ then $2 X_{i j}$ has log-concave distribution (i.e., for integers $u \geq 1$, $\left.(p(u))^{2} \geq p(u-1) p(u+1)\right)$. Proposition 1 and Corollary 2 (Ross, 2021) hold in our model with $p=0$ or $p \in[1 / 3,1)$, and therefore $\sum_{i=1, i \neq j}^{n} I_{i}^{(n)} \mid I_{j}^{(n)}=1$ is stochastically smaller than $\sum_{i=1, i \neq j}^{n} I_{i}^{(n)}$. Therefore, from the Corollary 2.C.2 (Barbour et al., 1992) we obtain (A1). Proof. (Assertion 2). Follows from Feller (1971)(p. 552-553, Theorem 2 or 3). 
Proof. (Assertion 3). Follows from Assertion 2 combined with Cramér (1946) result on page 374 of his book.

Proof. (Assertion 4). Recall that $s_{1}=X_{12}+X_{13}+\cdots+X_{1 n}$ and $s_{2}=X_{21}+X_{23}+\cdots+X_{2 n}$. Hence, condition on the event $X_{12}=k, k \in\{0,1 / 2,1\}, s_{1}$ and $s_{2}$ are independent. Let $s_{1^{\prime}}=X_{13}+\cdots+X_{1 n}, s_{2^{\prime}}=X_{23}+\cdots+X_{2 n}$ and denote by $s_{1^{\prime}}^{*}, s_{2^{\prime}}^{*}$ the corresponding normalized scores (zero expectation and unit variance). We have,

$$
\begin{aligned}
& P\left(s_{1}^{*}>x_{n}(t), s_{2}^{*}>x_{n}(t) \mid X_{12}=k\right)=P\left(s_{1}^{*}>x_{n}(t) \mid X_{12}=k\right) P\left(s_{2}^{*}>x_{n}(t) \mid X_{12}=k\right) \\
& =P\left(s_{1^{\prime}}^{*}>x_{n-1}(t) \frac{x_{n}(t)}{x_{n-1}(t)} \sqrt{\frac{n-1}{n-2}}-\frac{\sqrt{2}(k-1 / 2)}{\sqrt{n-2}}\right) \\
& P\left(s_{2^{\prime}}^{*}>x_{n-1}(t) \frac{x_{n}(t)}{x_{n-1}(t)} \sqrt{\frac{n-1}{n-2}}-\frac{\sqrt{2}((1-k)-1 / 2)}{\sqrt{n-2}}\right) \\
& \sim P\left(s_{1^{\prime}}^{*}>x_{n-1}(t)\right) P\left(s_{2^{\prime}}^{*}>x_{n-1}(t)\right) .
\end{aligned}
$$

Combining ( $(\underline{\mathrm{F} 3})$ with the formula of total probability we obtain

$$
P\left(s_{1}^{*}>x_{n}(t), s_{2}^{*}>x_{n}(t)\right) \sim P\left(s_{1^{\prime}}^{*}>x_{n-1}(t)\right) P\left(s_{2^{\prime}}^{*}>x_{n-1}(t)\right)
$$

and combining it with Assertion 3 we obtain (A4).

Remark 1. It remains an open problem if Theorem 1 holds also for $p \in(0,1 / 3)$.

\section{Asymptotic distribution of the order statistics of the normalized scores}

An immediate consequence of Theorem 1 is given below and describes the asymptotic distribution of the ordered normalized scores.

Result 1. For $p=0$ or $p \in[1 / 3,1)$ and $j=0,1 \ldots, n-1$,

$$
\lim _{n \rightarrow \infty} P\left(s_{(n-j)}^{*}<a_{n}+b_{n} t\right)=G(t)\left(1+e^{-t}+\cdots+e^{-j t} / j !\right)
$$

where $a_{n}$ and $b_{n}$ are defined in (1) and $G(t)=e^{-e^{-t}}$ ("Gumbel" distribution function). 
Proof. For $p=0$ or $p \in[1 / 3,1)$, Result 1 follows from Theorem 1, since $P\left(s_{(n-j)}^{*} \leq x_{n}(t)\right)=P\left(S_{n} \leq j\right)$, and therefore

$$
\lim _{n \rightarrow \infty} P\left(s_{(n-j)}^{*} \leq x_{n}(t)\right)=\lim _{n \rightarrow \infty} P\left(S_{n} \leq j\right)=e^{-e^{-t}} \sum_{k=0}^{j} \frac{e^{-t k}}{k !} .
$$

We demonstrate our results with the three best scores in Model M.

\subsection{Maximal Score}

For $p=0$ or $p \in[1 / 3,1)$, we obtain from Result 1 the following corollary.

\section{Corollary 1.}

$$
\begin{aligned}
& E\left(s_{(n)}\right) \sim \frac{n-1}{2}+\sqrt{\frac{(n-1) \log (n)(1-p)}{2}} \\
& +\sqrt{\frac{(n-1)(1-p)}{2 \ln (n)}}\left\{\frac{\gamma}{2}-\frac{1}{4}(\log \log (n)+\log (4 \pi))\right\} \equiv \hat{E}_{(n)}, \\
& \sigma\left(s_{(n)}\right) \sim \frac{\pi}{4 \sqrt{3}} \sqrt{\frac{(n-1)(1-p)}{2 \log (n)}} \equiv \hat{\sigma}_{(n)},
\end{aligned}
$$

where $\gamma=0.5772156649 \ldots$ is the Euler constant.

Proof. The moments under the distribution function $G$ can be obtained based on the fol-

lowing consideration. If $Y_{1}, \ldots, Y_{n}$ are independent $\exp (1)$ random variables, then straightforward calculation shows (see for example Grimmett and Stirzaker (2020)):

$$
\lim _{n \rightarrow \infty} P\left(Y_{(n)}-\log (n) \leq t\right)=G(t)
$$

and for $\mathrm{r}=0,1,2, \ldots, n$,

$$
(n+1-r)\left(Y_{(r)}-Y_{(r-1)}\right)
$$

are independent exponential random variables with rate parameter 1 , where $Y_{(0)}$ is defined as zero. Since

$$
Y_{(k)}=Y_{(1)}+\left(Y_{(2)}-Y_{(1)}\right)+\cdots+\left(Y_{(k)}-Y_{(k-1)}\right)
$$

we obtain that

$$
E\left(Y_{(n)}\right)=\sum_{j=1}^{n} \frac{1}{j}, \quad \operatorname{Var}\left(Y_{(n)}\right)=\sum_{j=1}^{n} \frac{1}{j^{2}}
$$


From $\lim _{n \rightarrow \infty}\left\{\sum_{j=1}^{n} \frac{1}{j}-\log n\right\}=\gamma, \lim _{n \rightarrow \infty} \sum_{j=1}^{n} \frac{1}{j^{2}}=\frac{\pi^{2}}{6}$ (see for example Courant and Robbins (1996)), and (2), we obtain the expectation and variance under the distribution function $G$ as $E_{G}=\gamma, \operatorname{Var}_{G}=\frac{\pi^{2}}{6}$. Combining this with Result 1, we have

$$
E\left(s_{(n)}^{*}\right) \sim \gamma b_{n}+a_{n}, \quad \sigma\left(s_{(n)}^{*}\right) \sim \sqrt{\frac{\pi^{2}}{6}} b_{n} .
$$

Then, upon substituting $s_{(n)}^{*}=\left(s_{(n)}-E_{n}\right) / \sigma_{n}$, Corollary 1 follows.

In the following table, we compare $E\left(s_{(n)}\right)$ with $\hat{E}_{(n)}$ and $\sigma\left(s_{(n)}\right)$ with $\hat{\sigma}_{(n)}$ in this manner: We fix $p=2 / 3$ and for $\mathrm{n}=10,20,50,100,1000$, and 10000 we evaluate $E\left(s_{(n)}\right)$ and $\sigma\left(s_{(n)}\right)$ using Monte-Carlo (MC) simulation. Values of $\hat{E}_{(n)}$ and $\hat{\sigma}_{(n)}$ obtained based on Corollary 1 .

\begin{tabular}{lllllll}
$\mathrm{n}$ & $E\left(s_{(n)}\right)$ & $\hat{E}_{(n)}$ & $\left|\hat{E}_{(n)} / E\left(s_{(n)}\right)-1\right| * 100 \%$ & $\sigma\left(s_{(n)}\right)$ & $\hat{\sigma}_{(n)}$ & $\left|\hat{\sigma}_{(n)} / \sigma\left(s_{(n)}\right)-1\right| * 100 \%$ \\
\hline 10 & 5.833 & 5.912 & 1.360 & 0.469 & 0.518 & 10.454 \\
20 & 11.89 & 11.944 & 0.456 & 0.627 & 0.659 & 5.189 \\
50 & 29.08 & 29.162 & 0.283 & 0.912 & 0.927 & 1.563 \\
100 & 56.73 & 56.843 & 0.199 & 1.219 & 1.214 & 0.426 \\
1,000 & 529.12 & 529.352 & 0.044 & 3.259 & 3.148 & 3.529 \\
10,000 & 5110.23 & 5111.295 & 0.0212 & 8.949 & 8.626 & 3.742
\end{tabular}

Table 1: The number of Monte-Carlo repetitions is 100,000 for $\mathrm{n}=10,20,50,100 ; 10,000$ for $\mathrm{n}=1000$; and 500 for $\mathrm{n}=10,000$.

\subsection{Second and third largest scores}

For $p=0$ or $p \in[1 / 3,1)$, we also obtain from Result 1 the following corollary.

\section{Corollary 2.}

$$
\begin{aligned}
& E\left(s_{(n-1)}^{*}\right) \sim \gamma b_{n}+a_{n}-b_{n}, \quad \sigma\left(s_{(n-1)}^{*}\right) \sim \sqrt{\left(\frac{\pi^{2}}{6}-1\right)} b_{n}, \\
& E\left(s_{(n-2)}^{*}\right) \sim \gamma b_{n}+a_{n}-3 / 2 b_{n}, \quad \sigma\left(s_{(n-2)}^{*}\right) \sim \sqrt{\left(\frac{\pi^{2}}{6}-1.25\right)} b_{n}
\end{aligned}
$$


Proof. From Theorem 2.2.2 in Leadbetter et al. (1983), we obtain the following result: if $Y_{1}, \ldots, Y_{n}$ are independent $\exp (1)$ random variables, then for $j=1,2$

$$
\lim _{n \rightarrow \infty} P\left(Y_{(n-j)}-\log (n) \leq t\right)=G(t)\left(1+e^{-t}+\cdots+e^{-j t} / j !\right)
$$

The rest of the proof is similar to the proof of Corollary 1.

Substituting $s_{(j)}^{*}=\left(s_{(j)}-E_{n}\right) / \sigma_{n}$ for $j=n-1, n-2$, we obtain the values $\left.E\left(s_{(j)}\right), \sigma\left(s_{(j)}\right)\right), \widehat{E}_{(j)}, \widehat{\sigma}_{(j)}$, which are similar to the corresponding values obtained in Corollary 1 for the case $j=n$. In the case where $p=2 / 3$, we provide numerical comparisons for the second and third largest scores in a similar manner as was done in Table 1.

\begin{tabular}{lllllll}
$\mathrm{n}$ & $E\left(s_{(n-1)}\right)\left(\sigma\left(s_{(n-1)}\right)\right)$ & $\widehat{E}_{(n-1)}\left(\widehat{\sigma}_{(n-1)}\right)$ & $E\left(s_{(n-2)}\right)\left(\sigma\left(s_{(n-2)}\right)\right)$ & $\widehat{E}_{(n-2)}\left(\widehat{\sigma}_{(n-2)}\right)$ & $r_{(n-1)}$ & $r_{(n-2)}$ \\
\hline 10 & $5.400(0.338)$ & $5.509(0.324)$ & $5.093(0.273)$ & $5.307(0.254)$ & 2.009 & 4.195 \\
20 & $11.305(0.446)$ & $11.43(0.413)$ & $10.95(0.374)$ & $11.173(0.323)$ & 1.106 & 2.037 \\
50 & $28.277(0.649)$ & $28.44(0.580)$ & $27.816(0.541)$ & $28.079(0.454)$ & 0.576 & 0.946 \\
100 & $55.695(0.858)$ & $55.896(0.760)$ & $55.113(0.712$ & $55.423(0.595)$ & 0.361 & 0.563 \\
1,000 & $526.48(2.154)$ & $526.9(1.971)$ & $525.05(1.764)$ & $525.67(1.543)$ & 0.080 & 0.118 \\
10,000 & $5103.2(5.866)$ & $5104.6(5.401)$ & $5099.5(4.672)$ & $5101.2(4.227)$ & 0.027 & 0.033
\end{tabular}

Table 2: The number of Monte-Carlo repetitions is 100,000 for $\mathrm{n}=10,20,50,100 ; 10,000$ for $\mathrm{n}=1000$; and 500 for $\mathrm{n}=10,000 ; r_{(j)}=\left|\widehat{E}\left(s_{(j)}\right) / E\left(s_{(j)}\right)-1\right| * 100 \%, j=n-1, n-2$.

\section{Acknowledgement}

I thank Abram Kagan for describing a score issue in chess round-robin tournaments with draws. I am grateful to Pavel Chigansky for pointing out my mistake in Malinovsky (2021a). It led to exciting research that resulted in a correction (Malinovsky, 2021b) and is presented in this version. I also thank Sheldon Ross for referring to his recent paper that turned out to be essential in the proof of the main result in this work. This research was supported by grant no 2020063 from the United States-Israel Binational Science Foundation (BSF). 


\section{References}

Barbour, A. D., Holst, L., Janson, S. (1992). Poisson approximation. Oxford Studies in Probability. The Clarendon Press, Oxford, New York.

Cramér, H. (1946). Mathematical Methods of Statistics. Primceton University Press.

Courant, R., and Robbins, H. (1996). What Is Mathematics? An Elementary Approach to Ideas and Methods. Oxford University Press 2nd edition reviewed by Ian Stewart.

Feller, W. (1971). An introduction to probabilty theory and its applications. Vol. II. Second edition. New York-London-Sydney: Wiley.

Grimmett, G. R. and Stirzaker, D R. (2020). Probability and random processes. 4th Edition. Oxford University Press.

Huber, P. J. (1963). A remark on a paper of Trawinski and David entitled: Selection of the best treatment in a paired comparison experiment. Ann.Math.Statist. 34, 92-94.

Leadbetter, M. R., Lindgren, G., Rootzén, H. (1983). Extremes and related properties of random sequences and processes. Springer Series in Statistics. Springer-Verlag, New York-Berlin.

Malinovsky, Y.(2021a). On the distribution of winners' scores in a round-robin tournament. Prob. in Eng. and Inf. Sciences.DOI: https://doi.org/10.1017/S0269964821000267. In press.

Malinovsky, Y. (2021b). Correction to "On the distribution of winners' scores in a roundrobin tournament." Prob. in Eng. and Inf. Sciences. In press from December 9, 2021.

Malinovsky, Y., Moon, J. W. (2021). On the negative dependence inequalities and maximal score in round-robin tournament. https://arxiv.org/abs/2104.01450.

Moon, J. W. (2013). Topics on Tournaments. [Publicaly available on website of Project Gutenberg https://www .gutenberg .org/ebooks/42833.

Ross, S. M. (2021). Team's seasonal win probabilities. Prob. in Eng. and Inf. Sciences. DOI: https://doi.org/10.1017/S026996482100019X. In press. 